\title{
BMJ Open Interprofessional evidence-based counselling programme for complementary and integrative healthcare in patients with cancer: study protocol for the controlled implementation study CCC-Integrativ
}

Jan Valentini (D) , ${ }^{1}$ Daniela Fröhlich, ${ }^{1}$ Regina Stolz, ${ }^{1}$ Cornelia Mahler, ${ }^{2}$ Peter Martus, ${ }^{3}$ Nadja Klafke, ${ }^{4}$ Markus Horneber, ${ }^{5}$ Jona Frasch, ${ }^{6}$ Klaus Kramer, ${ }^{7}$ Hartmut Bertz, ${ }^{8}$ Barbara Grün, ${ }^{9}$ Katrin Tomaschko-Ubeländer, ${ }^{10}$ Stefanie Joos, ${ }^{1}$ CCC-Integrativ study group

To cite: Valentini J, Fröhlich D, Stolz R, et al. Interprofessional evidence-based counselling programme for complementary and integrative healthcare in patients with cancer: study protocol for the controlled implementation study CCC-Integrativ. BMJ Open 2022;12:e055076. doi:10.1136/ bmjopen-2021-055076

- Prepublication history for this paper is available online. To view these files, please visit the journal online (http://dx.doi. org/10.1136/bmjopen-2021055076).

Received 21 July 2021 Accepted 13 January 2022

D) Check for updates

(C) Author(s) (or their employer(s)) 2022. Re-use permitted under CC BY-NC. No commercial re-use. See rights and permissions. Published by BMJ.

For numbered affiliations see end of article.

Correspondence to Dr Jan Valentini; jan.valentini@med.unituebingen.de

\section{ABSTRACT}

Introduction According to international literature, patients with cancer wish to have information on complementary and integrative healthcare $(\mathrm{ClH})$. Medical guidelines recommend actively approaching patients with cancer discussing potential benefits and risks of individual $\mathrm{CIH}$ methods. While some $\mathrm{CIH}$ methods, for example, acupuncture and yoga, have been proven effective in high-quality studies, other $\mathrm{CIH}$ methods lack studies or bear the risk of interactions with chemotherapeutics, for example, herbal drugs. Therefore, an evidence-based interprofessional counselling programme on $\mathrm{ClH}$ will be implemented at four Comprehensive Cancer Centres in the federal state of Baden-Wuerttemberg, Germany.

Methods and analysis A complex intervention consisting of elements on patient, provider and system levels will be developed and evaluated within a multilayer evaluation design with confirmatory evaluation on patient level. Patients with a cancer diagnosis within the last 6 months will receive three individual counselling sessions on $\mathrm{CIH}$ within 3 months (=intervention on patient level). The counselling will be provided by an interprofessional team of medical and nursing staff. For this purpose, an intensive online training programme, a CIH knowledge database and an interprofessional team-building process were developed and implemented (=intervention on provider level). Moreover, training events on the basics of $\mathrm{ClH}$ are offered in the outpatient setting (=intervention on system level). Primary outcome of the evaluation at the patient level is patient activation measured (PAM) with the PAM13 after 3 months. Secondary outcomes, for example, quality of life, self-efficacy and clinical parameters, will be assessed at baseline, after 3 months and at 6 months follow-up. The intervention group $(n=1000)$ will be compared with a control group $(n=500$, treatment as usual, no $\mathrm{ClH}$ counselling. The outcomes and followup times in the control group are the same as in the intervention group. Moreover, the use of health services will be analysed in both groups using routine data. A
Strengths and limitations of this study

- For the first time, a transsectoral, interprofessional, evidence-based counselling programme for Complementary and Integrative Healthcare will be implemented at Comprehensive Cancer Centres Baden-Wuerttemberg, Germany.

- The complex evaluation will be conducted at the patient, provider and system levels within a controlled design.

The guiding (confirmatory) hypothesis is at the patient level measured by patient activation (Patient Activation Measure-13).

- Randomisation at the patient level is not possible due to the naturalistic study design.

- On the provider level, a training programme for the counselling team is designed and evaluated as blended-learning programme with online (asynchronous) and onsite (synchronous) formats.

qualitative-quantitative process evaluation as well as a health economic evaluation will identify relevant barriers and enabling factors for later roll-out.

Ethics and dissemination The study has been approved by the appropriate Institutional Ethical Committee of the University of Tuebingen, No. 658/2019B01. The results of these studies will be disseminated to academic audiences and in the community.

Trial registration number DRKS00021779; Pre-results.

\section{INTRODUCTION}

According to previous international studies, there is a high use of naturopathic/complementary approaches (complementaryand integrative healthcare, $\mathrm{CIH}$ ) among patients with cancer. A meta-analysis shows that about 
$40 \%$ of all oncology patients use $\mathrm{CIH}^{1}$; for patients with breast cancer, up to $80 \%$ can be assumed. ${ }^{2}$ For some $\mathrm{CIH}$ methods, positive effects in patients with cancer have been shown in randomised controlled trials (RCT) and meta-analyses, for example, meditation, particularly mindfulness-based stress reduction for mood disturbance and depression, Yoga and Tai Ji for improving quality of life and fatigue, acupuncture and acupressure to reduce nausea and pain, individual phytotherapeutics and herbal medicines such as ginger for nausea ${ }^{3-5}$ In addition, many of these CIHs have the potential to empower and activate patients as well as improve self-efficacy. ${ }^{6}$ The promotion of patient activation, ${ }^{7}$ self-management strategies ${ }^{8}$ and health literacy ${ }^{9}$ have been shown to empower patients with cancer ${ }^{10}$ and contribute to reducing the use of health services. ${ }^{11}$ Thereby, patient activation is considered as an overarching concept including knowledge, skill and confidence for self-management in chronic diseases. ${ }^{12}$

However, CIH also entails clinical risks; for example, phytotherapeutics and micronutrients, especially in high doses (vitamins, selenium), can interact with chemotherapy. ${ }^{13}{ }^{14} \mathrm{~A}$ further risk arises as patients often seek help outside conventional healthcare structures (eg, alternative practitioners) with unforeseeable health consequences such as delayed diagnosis and failure to provide indicated treatments. ${ }^{15} 16$ In addition, there are risks for economic harm as the majority of costs for CIH procedures are not covered by public insurance. ${ }^{17}$ Between $20 \%$ and $77 \%$ of patients with cancer do not inform their treating physicians when using $\mathrm{CIH} .{ }^{18-20}$ This lack of communication may endanger the doctor-patient relationship and contribute to the discontinuation of conventional therapy. ${ }^{152} 22$

Patient-centred care for cancer includes supportive measures that enable patients to cope as well as possible with their diagnosis and therapy, including its side effects. ${ }^{23-25}$ These supportive measures may also include CIH methods. Therefore, according to current German S3 guidelines on breast cancer, cervical cancer, ovarian cancer, as well as the guidelines on complementary medicine in the treatment of oncology patients, ${ }^{3}$ palliative medicine and psycho-oncology, there is a consensus recommendation that all patients should be asked about their need for information on their use of $\mathrm{CIH}$ procedures. ${ }^{32}$ However, this recommendation contrasts with a lack of human resources in oncologic clinics and insufficient CIH knowledge among medical and nursing staff. Therefore, this recommendation cannot be implemented properly in everyday care, and the topic of CIH is often not sufficiently or not at all addressed in patient communication. The aim of the Comprehensive Cancer Centres (CCC)-Integrativ study is to develop and evaluate a complex intervention as defined by elements on patient, provider and system levels to improve $\mathrm{CIH}$ for patients with cancer. In detail, we aim to evaluate whether interprofessional counselling about CIH improves patient activation and patients' confidence in contributing to their health.

\section{METHODS AND ANALYSIS}

\section{Theoretical framework and objectives}

The project pursues a health services research approach, and therefore, objectives and interventions will be differentiated in relation to the patient level, provider level and system level or-seen from a health services research perspective-in relation to microlevel, mesolevel and macrolevel.

On the patient (micro) level, the project aims to activate patients and promote their self-efficacy (see figure 1). It is expected that by enhanced empowerment, the patient's quality of life and clinical outcomes will be improved, which can, in turn, lead to reduced use of health services in the further course. ${ }^{27}$

Objectives at the provider (meso) level are an improvement of knowledge and communication skills regarding $\mathrm{CIH}$ as well as improved job satisfaction and interprofessional collaboration between doctors and nurses within the care setting.

At the system (macro) level, the objectives include cost reduction and a transsectoral increase in knowledge of CIH (see figure 2).

\section{Setting and study design}

Within the CCC-Integrativ study, an evidence-based interprofessional counselling programme on $\mathrm{CIH}$ for oncology patients will be implemented and evaluated at the four CCC (Freiburg, Heidelberg, Tuebingen-Stuttgart, Ulm)

\section{INTERVENTION}

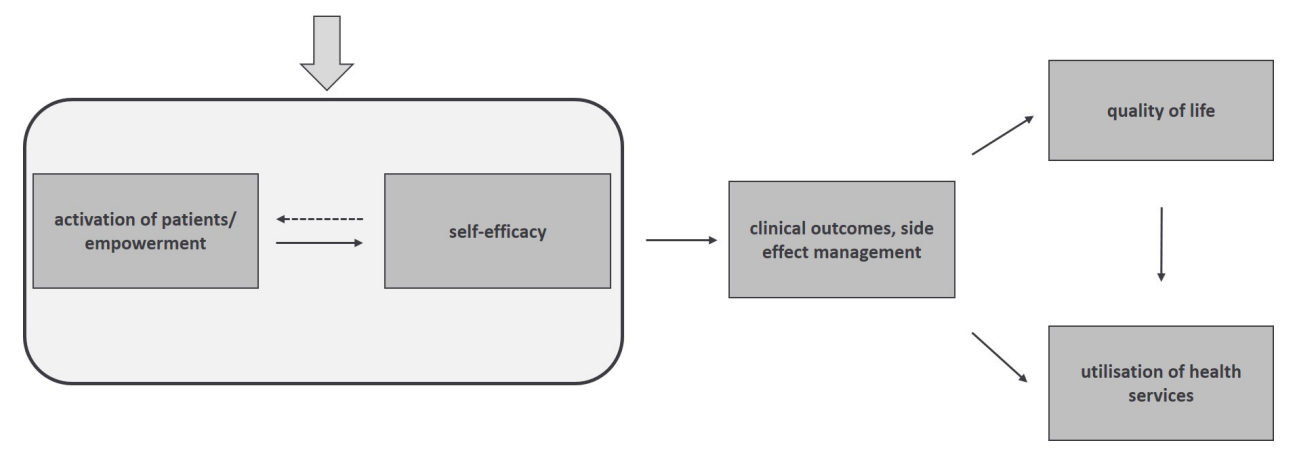

Figure 1 Theoretical model for outcomes on patient level. 
Levels

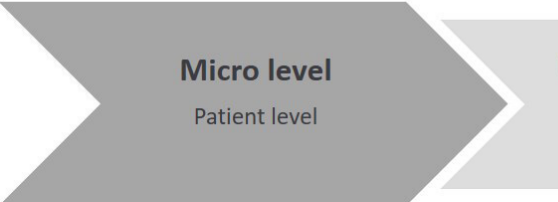

Intervention

Objectives
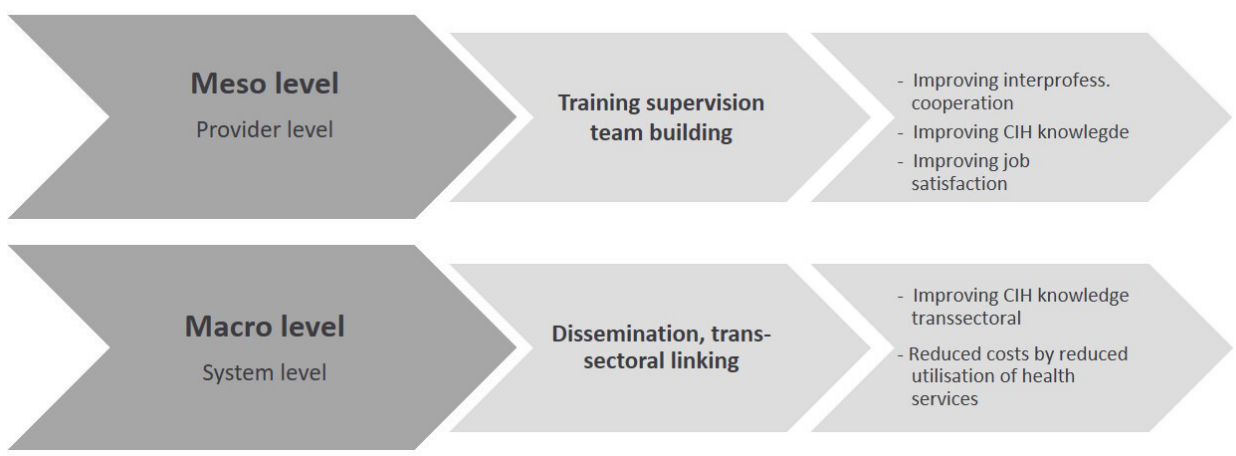

Figure 2 Outcomes framework for CCC-Integrativ. CCC, Comprehensive Cancer Centres; CIH, complementary and integrative healthcare.

in Baden-Wuerttemberg. CCC are implemented at most university hospitals in Germany to ensure a high standard of medical care for oncology patients and are considered centres of excellence in oncology.

As described above, CCC-Integrativ will be evaluated at patient, provider and system levels, whereby the confirmatory testing will be on patient level. The accompanying process evaluation, which also analyses aspects of the microlevel, mesolevel and macrolevel, is presented in detail in a separate protocol.

The total study duration is 36 months. For the individual study participant, the study lasts 6 months, including the follow-up survey (intervention group and control group). The preparation period runs from months 1-6, the primary data collection in the control group from months 7 to 18 (recruitment months 7-9). Data collection in the intervention group from months 13-30 (recruitment months 13-24).

The intervention group is compared with a control group and a reference group. The control group is recruited 6 months before the start of the intervention phase. Identical outcome parameters are collected. The reference group results from claims data of the statutory health insurance of the Allgemeine Ortskrankenkasse Baden-Wuerttemberg (AOK BW) without additional primary data collection. The reference group allows us to examine the representativity of the study population compared with the target population (members of AOK$\mathrm{BW}$ ). If differences between the control group and the reference group are detected, that is, study effects are present, these will be accounted for in the comparison of the reference group with the intervention group to disentangle study effects and intervention effects.

A classical parallel-group design with randomisation at the patient level did not seem feasible, as previous studies have shown that patients with high use of or need for counselling on $\mathrm{CIH}$ cannot be randomised. ${ }^{24}$
Also, cluster randomisation had to be rejected due to possible contamination problems. Given that all four participating CCCs are located in the same federal state (Baden-Wuerttemberg, Germany) and thus within a few hours' drive, we could not exclude that patients treated in one CCC would seek counselling in another participating CCC. Thus, we decided to choose different time intervals within the study period for control and intervention patients.

\section{Intervention on patient level}

The intervention consists of an interprofessional, evidence-based counselling service for patients with counselling needs in the field of CIH. Key points of the counselling are information giving and guidance for health needs in the context of CIH to increase patient activation and self-efficacy.

The counselling concept takes into account the patient's resources, integrates conventional healthcare (as provided by the CCCs in routine care) and provides information on $\mathrm{CIH}$ and care services with CIH. The central point of the counselling is the specific CIH counselling needs of the patient (see figure 3). In addition to the specific patient needs, nutrition, exercise and stress management are addressed as resources. If there is a need for further counselling on these three topics or $\mathrm{CIH}$ counselling sessions were to bring up psychological distress for any patients, referrals are made to the specific counselling services for nutrition, exercise and psychooncology offered in all participating CCCs.

The CIH counselling service is provided by interprofessional teams consisting of specially trained physicians and nurses at each of the four participating CCCs and is integrated into the existing structures at the four participating CCCs in Freiburg, Heidelberg, TuebingenStuttgart und Ulm. Our concept for counselling follows 


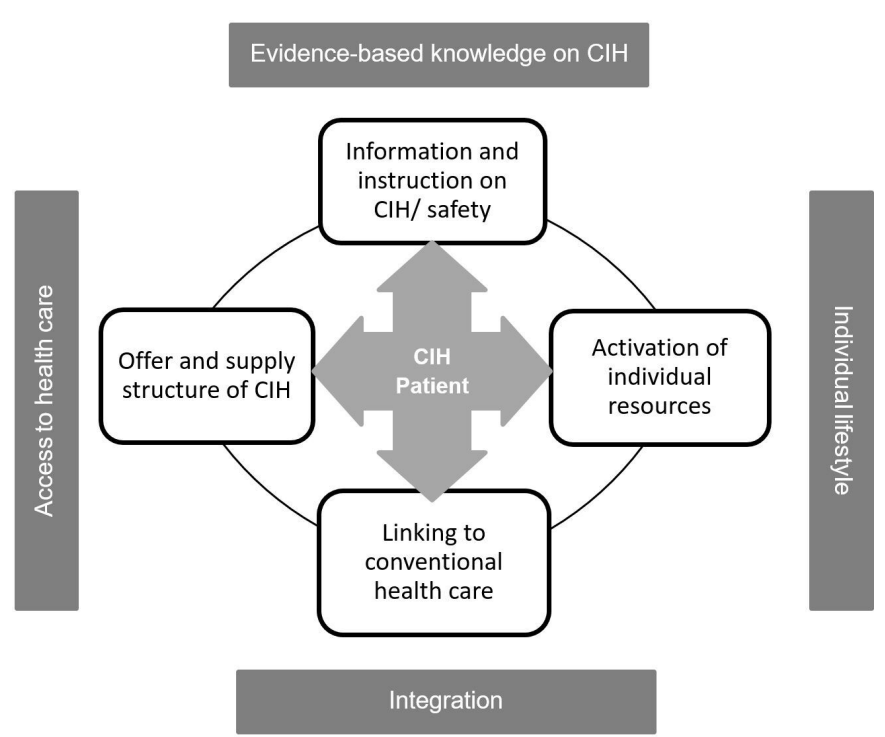

Figure 3 CCC-Integrativ counselling model of the intervention. CCC, Comprehensive Cancer Centres; $\mathrm{CIH}$, complementary and integrative healthcare.

an evidence-based approach, integrating patient preferences and individual medical and nursing expertise.

\section{Intervention and control group}

\section{Intervention group}

Patients of the intervention group will be offered interprofessional individual counselling. Eligible patients will receive at least three counselling sessions on evidencebased nursing and/or medical interventions within 3 months. The first counselling session will be face-toface and interprofessional and is planned for a duration of $60 \mathrm{~min}$. The subsequent two counselling sessions may be monoprofessional and performed by telephone or as video counselling with a duration of approximately $30 \mathrm{~min}$.

If required within a counselling session, easy to understand information leaflets developed in the preparation phase for the most relevant symptoms and CIH methods and/or prescriptions for phytotherapeutics are handed out to patients to promote patient activation. In case of recommendation of external applications, these can also be shown to the patient on-site. Furthermore, counselling on where to find trustworthy information about $\mathrm{CIH}$ on the internet as well as how to evaluate CIH providers and CIH products will be provided.

To standardise the counselling process, a structured guideline for the consulting teams is developed before the intervention starts and will be practised in the training programme.

\section{Control group and reference group}

The control group and the reference group receive conventional healthcare as usual provided by the CCCs (treatment as usual).
Participants

Eligible participants consist of outpatient oncology patients at the four CCC (Tuebingen-Stuttgart, Ulm, Freiburg and Heidelberg) of the Federal State of BadenWuerttemberg, Germany).

\section{Inclusion and exclusion criteria}

The following inclusion criteria apply to the intervention group and the control group:

- Diagnosis of cancer including progression or recurrence within the last 6 months (all cancer types can be included).

- The patient must be able to attend counselling on site.

- Treatment at one of the participating CCCs or consultation for a second opinion.

- Age $\geq 18$ years.

- Need for CIH counselling (attested by actively contacting the local counselling centre by email, phone or in-person).

- A present signed declaration of consent to the study and to data protection (Informed consent).

Exclusion criteria:

- For intervention group: participation in the control group.

- Language or cognitive impairments preventing patients from completing questionnaires independently.

\section{Recruitment}

\section{Recruitment procedure in the intervention group}

Information materials on the project (as flyers, brochures and a website) are displayed in outpatient clinics, day clinics and the other counselling centres at the four CCCs. In addition, the project will be presented at other counselling services, support groups and established formats (senior physician conferences, nursing service meetings, etc) in each CCC. In the preparatory phase, information events will also be held at each CCC to report on the project. Patients are to become aware of the counselling via flyers, newspaper reports, word-of-mouth information, via the treating physicians and via being actively approached by the study staff.

\section{Recruitment procedure in the control group}

Patients in the control group are actively approached by study staff (doctors, nurses, study assistants) during waiting times in the rooms of the CCC of the day clinic. The patients are informed about the study and asked whether they would enter the control group. The time points of data collection as well as the instruments used to collect the primary and secondary outcomes correspond to those in the intervention group (T1, T2, T3). Since the counselling service is not yet established, inclusion in the intervention group is not yet possible. As compensation for their time, the patients are offered the CIH counselling service after the end of the observation period of 6 months (outside the study setting). 
Recruitment procedure in the reference group

As there is no primary data collection in the reference group, no recruitment is needed. Instead, secondary data from the AOK-BW will be used in pseudonymised form.

\section{Intervention on provider level}

Blended-learning training programmes for counselling teams

The training programme for counselling teams is designed as blended-learning with online (asynchronous) and onsite (synchronous) formats such as webinars or faceto-face format. The contents of the training programme were developed during the preparatory phase of the project based on existing guidelines and expertise from two previous studies: the CONGO study and the project KOKON. Data from KOKON (www.kompetenznetzkokon.de) show the specific information that doctors need for good counselling ${ }^{28}$ and how this can be implemented successfully, ${ }^{29}$ whereas CONGO focused on nursing applications in the context of supportive cancer care. ${ }^{3031}$ The online content of CCC-Integrativ is presented through an online learning management system (ILIAS-software). Training consists of evidence-based text material, live lectures and asynchronous lectures on individual CIH methods and of communication training. For the latter, training with simulation patients was integrated into the synchronous online formats. The structure and overall contents of the blended-learning training programme will be presented in detail in a separate publication (in preparation).

\section{Tool box for counselling teams}

In order to achieve standardised and evidence-based counselling on $\mathrm{CIH}$, specific symptom-driven guidelines for the most relevant symptoms (eg, on chemotherapyinduced nausea and vomiting) are developed on the basis of structured literature research and expert consensus process. Altogether, 20 symptom-driven guidelines will be developed to provide a basic counselling source of information for the counselling teams.

\section{Knowledge database on $\mathrm{CIH}$}

Within the KOKON Project (KOKONbase), a knowledge database on clinical efficacy and safety of complementary medicine in oncology was implemented. The contents of the database are linked to the international information portal of the CAM Cancer Project ${ }^{32}$ and include free access via Onkopedia (https://www.onkopedia.com/de), the guideline portal of the German Society for Haematology and Medical Oncology. During the CCC-Integrativ Study, the database will be constantly updated and supplemented with content on complementary nursing.

\section{Interprofessional team building}

Interprofessional counselling requires interprofessional team building of the counselling teams (nurse and physician). Therefore, workshops to promote interprofessional collaboration using the TEAM ${ }^{\mathrm{c}}$ approach $^{33} 34$ are performed for each counselling team, either onsite or online (synchronous) and complement the blendedlearning programme.

\section{Intervention on system level}

As part of the system-level intervention, basic training on $\mathrm{CIH}$ is provided to healthcare professionals on a crosssectoral level, ranging from university hospitals to primary care. The target groups are, for example, health workers in the CCCs, general practitioners and ambulatory care services. The aim of this training is to achieve a common understanding of the term $\mathrm{CIH}$, discuss possible indications for the application and potential risks of $\mathrm{CIH}$ based on scientific literature and to address reliable sources of information on CIH. The number of training sessions and the exact target persons are not fixed in advance but will be determined by the demand of the different groups of healthcare professionals during the intervention phase.

\section{Primary and secondary outcomes on patient level Primary outcome}

The primary outcome parameter for assessing the intervention effect is patient activation, operationalised by the Patient Activation Measure questionnaire of the German version (PAM-13-D) after 3 months (T2) ${ }^{735}$ The PAM-13 is widely used internationally, also within the oncology setting, and has been validated in German. It has been shown to be a valid and reliable predictor of patient activation. ${ }^{35} 36$ The PAM-13 measures the extent to which a patient actively participates in his or her treatment. Furthermore, it measures the patient's active role in managing his/her own health and the extent to which he/she feels competent to fulfil that role. This construct includes aspects of health and patient knowledge, skill and confidence for self-management and therefore allows us to omit other concept-specific questionnaires, reducing the patient's overall burden of filling out questionnaires. A correlation between a higher level of patient activation (measured by the PAM-13) and better health outcomes, improved treatment adherence and a reduction in healthcare costs has been shown. ${ }^{37}$ The PAM-13-D is completed by the patients themselves. In the control and the intervention groups, PAM-13-D will be measured at baseline (T1), after 3 months (T2) and 6 months follow-up (T3) according to table 1.

\section{Secondary outcomes}

One part of the secondary outcomes at patient level is collected via primary data collection; another part is based on routine data. Secondary outcomes via primary data collection include quality of life, self-efficacy, depression, fatigue, symptom management, health literacy and healthcare utilisation (HCU). All outcomes will be measured with validated instruments and assessed at baseline (T1), after 3 months (T2) and 6 months follow-up (T3) as described in table 1.

The following clinical parameters, not shown in table 1, are also collected at patient level (survey dates are given in brackets): diagnosis including the Classification of 
Table 1 Primary and secondary outcomes at patient level in the intervention and control group

\section{patients}

\begin{tabular}{|c|c|c|c|c|c|}
\hline Outcomes & Instrument & Items & $\begin{array}{l}\text { T1 } \\
\text { Baseline }\end{array}$ & $\begin{array}{l}\text { T2 } \\
3 \text { months }\end{array}$ & $\begin{array}{l}\text { T3 } \\
6 \text { months follow-up }\end{array}$ \\
\hline \multicolumn{6}{|l|}{ Primary outcome } \\
\hline \multicolumn{6}{|l|}{ Secondary outcomes } \\
\hline Health status & EQ-5D & 5 & $x$ & $x$ & $x$ \\
\hline Quality of life & $\begin{array}{l}\text { EORTC-QLQ-C30 } \\
\text { (only question 29+30) }\end{array}$ & 2 & $\mathrm{x}$ & $x$ & $x$ \\
\hline Depression, agitation & PHQ-9 & 9 & $x$ & $\mathrm{x}$ & $\mathrm{x}$ \\
\hline Fatique & EORTC-QLQ-FA12 & 12 & $x$ & $x$ & $x$ \\
\hline Healthcare Utilisation Questionnaire & HCU-Q (adapted) & 11 & $x$ & $x$ & $x$ \\
\hline Sociodemographic data & & 26 & $\mathrm{x}$ & & \\
\hline Medical data & & 10 & $x$ & $x$ & $\mathrm{x}$ \\
\hline
\end{tabular}

EORTC-QLQ-C30, European Organisation for Research and Treatment of Cancer - Quality of Life of Cancer Patients Questionnaire - Core; EORTC-QLQ-FA12, European Organization for Research and Treatment of Cancer - Quality of Life of Cancer Patients Questionnaire - Cancer Related Fatigue; EQ-5D, European Quality of Life 5 Dimensions; HCU, Healthcare Utilisation Questionnaire; MIDOS2, Minimal Documentation System; MYCaW, Measure Yourself Concerns and Wellbeing; NEQ, Needs Evaluation Questionnaire; PAM-13, Patient Activation Measure 13; PHQ-9, Patient Health Questionnaire; SES6G, Self-Efficacy for Managing Chronic Disease 6-Item Scale; SILS, Single Item Literacy Screener.

Malignant Tumors (TNM) (T1), date of primary diagnosis of cancer (T1), if applicable, diagnosis of recurrence/ progression (T1, T2, T3), ongoing or planned oncological therapies (chemotherapy, surgery, etc) (T1, T2, T3).

Secondary outcomes based on routine data are use of inpatient and outpatient healthcare services, prescription of drugs, days of incapacity to work.

Routine (=claims) data are collected for billing purposes from the medical insurance company - the AOK BW. The AOK BW is the fifth largest health insurance fund in Germany and the largest in Baden-Wuerttemberg, with approximately 4.5 million insured persons. These data come from all AOK BW-insured persons who had received an International Statistical Classification of Diseases and Related Health Problems (ICD-10) cancer diagnosis according to the ICD-10 in the relevant time periods.

\section{Sample size}

Consistent data on the distribution of the primary endpoint PAM-13 is found in the literature. In a paper by Rademakers et al, ${ }^{38}$ sample size and SEs are each given for a Danish, a Dutch, a German and a Norwegian sample with a total of approx. 3500 patients. Back-calculation to the SD yields values between 14.49 and 14.61. In a study by Bates $e t a l,{ }^{39}$ SD between 14.3 and 14.8 were found. We, therefore, conservatively assume a SD of 15 in our planning. In the first study mentioned, a difference of 6.5 points was seen under intervention (68.5 vs 75.0).
With a study effect in the control group (eg, caused by using the instrument twice) of $30 \%$ (=1.95 points), we could expect a difference of 4.55 points. Conservatively assuming a difference of 4 points and the already justified $\mathrm{SD}$ of 15 points in the main outcome parameter PAM-13 between intervention and control group, a sample size of 669 patients is calculated (type 2 error of 0.10 and ratio of 2:1 between intervention $n=446$, and control group, $\mathrm{n}=223)$. Assuming a drop-out of $30 \%, 638$ patients must be included in the intervention group, and 319 must be included in the control group. For pragmatic considerations regarding the implementation character of the study (existing staff, established structures), we aim to recruit 1000 patients in the intervention group and 500 patients in the control group (see figure 4). With a sample size of 1500 patients $(1000+500)$ minus $30 \%$ dropouts, a group difference of 3.2 points on the PAM-13 would have a power of $90 \%$. By adjusting the baseline in a covariance analysis, a degree of freedom is lost, but it can be assumed that the power should be even higher due to the reduction in dispersion after adjustment.

For the reference group, health insurance data will be used.

\section{Data analysis}

Electronic data collection from the patients' questionnaires is recorded using Research Electronic Data Capture, a browser-based software for clinical and 

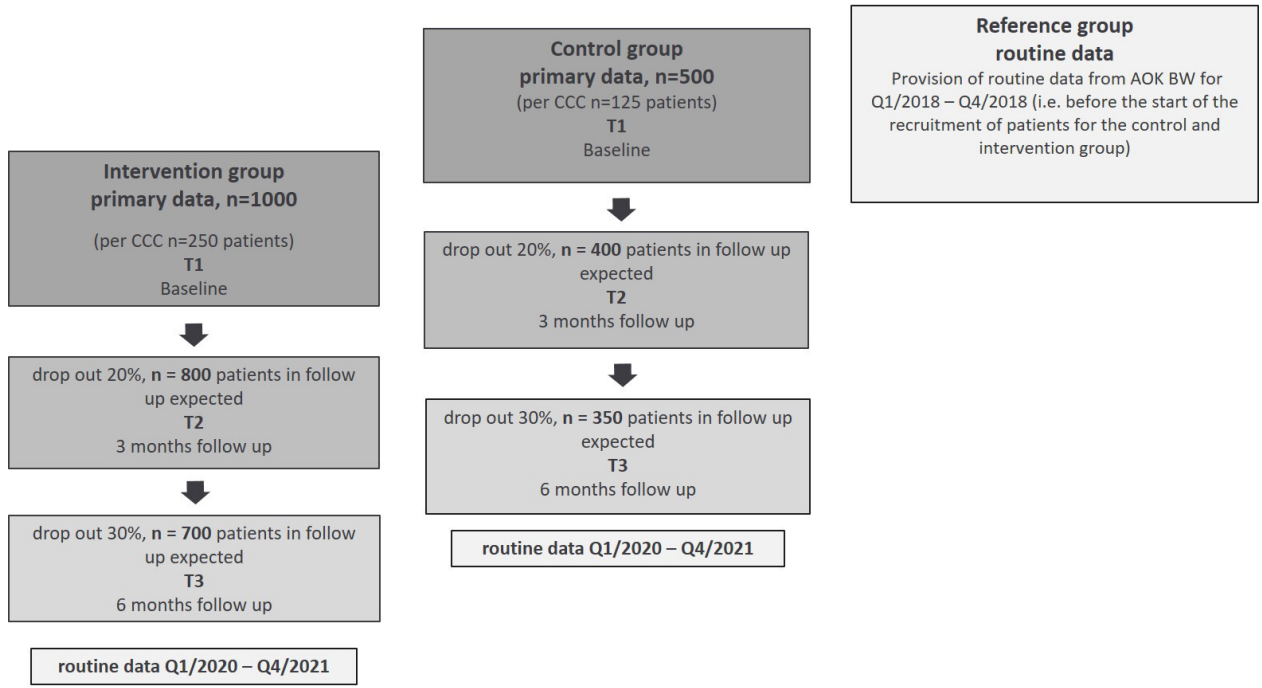

routine data Q1/2020 - Q4/2021

routine data Q1/2020 - Q4/2021

Figure 4 Flow chart data collection (T1-T3)/ routine data. AOK BW, Allgemeine Ortskrankenkasse Baden-Wuerttemberg; CCC, Comprehensive Cancer Centres.

translational research. ${ }^{40}$ All quantitative data will be transformed and imported into SPSS Version 28 or higher for further statistical analysis.

\section{Analysis of primary data outcomes}

The primary outcome parameter for the comparison of the intervention group with the control group is patient activation, which is measured by the PAM-13 after 3 months. For the primary outcome analysis of covariance will be applied with baseline adjustment and adjustment for study centre as a categorical variable. Secondary analyses of the primary endpoint include an additional analysis of covariance adjusted for age (quantitative), sex, diagnosis and disease stage, as well as a mixed model for $\mathrm{T} 1, \mathrm{~T} 2$ and T3 to analyse the temporal trend and test for maintenance effects at T3. Quantitative secondary endpoints will be analysed analogously to PAM, categorical data will be analysed using identically adjusted logistic regression models. For analyses of the secondary outcomes, $p$ values are reported but are not to be interpreted as confirmatory. The primary analysis population will be the intent to treat a population which includes all subjects with a baseline assessment. Multiple imputation methods are used to replace missing values. Imputation will be based on baseline values. Confirmatory interim analyses are not planned. The level of significance will be 0.05 (two sided).

\section{Analysis of routine data outcomes}

Routine data outcomes as described above will be compared between the intervention, the control and the reference group. In a first step, routine data outcomes of the control group will be compared with the reference group to assess possible selection bias due to the fact that study patients are all treated at the CCCs. In a second step, routine data outcomes of the intervention group are compared with the control group with adjustments derived from the first step comparison.

\section{Process evaluation}

In addition to the analyses mentioned above, a detailed qualitative-quantitative process evaluation will be conducted. This process evaluation is based on the Consolidated Framework for Implementation Research, a recognised framework in implementation research. ${ }^{41}$ In addition to evaluating the perspectives of patients and providers on the counselling sessions, the process evaluation aims to identify significant barriers and facilitate factors for successful implementation and later transferability of the intervention into standard care. All details regarding the concept and planned analyses can be found in a separate publication (in preparation).

\section{Health economic evaluation}

To evaluate the intervention's efficiency, a costeffectiveness and cost-utility analysis will be performed. To that end, the intervention and control groups will be compared on both the absolute and incremental costs and effects of care. The main analysis is conducted from the perspective of the insurees of the statutory health insurances, meaning that costs and effects incurred by the statutory health insurance as well as by the patients themselves are counted. A societal perspective will be considered in a sensitivity analysis. On the cost side, the intervention specific costs, the costs of inpatient and outpatient care, the costs of prescription drugs, therapeutic remedies, aids, informal care, productivity losses and $\mathrm{CIH}$ are considered. Data are collected using an adapted version of the HCU Questionnaire (HCU-Q) ${ }^{42}$ and supplemented for part of the intervention and control group with information from the AOK BW routine data (billing data). Using an incremental cost-effectiveness ratio, the efficiency of the intervention is quantified. This enables statements to be made about the programme's incremental costs per increment in quality of life and patient activation. Data from the reference group supplement and validate the 
data and analyses used and help contextualise the results. The time horizon of the analysis is 6 months. Both costs and effects are discounted at $3 \%$ p.a. In a deterministic sensitivity analysis, discounting rates of both $0 \%$ and $5 \%$ will be applied. Uncertainty will be accounted for in the sensitivity as well as subgroup analyses.

\section{Trial status}

Recruitment of the control group $(\mathrm{n}=502)$ was successfully completed in January 2021. The counselling for the intervention group started in January 2021. Lastpatient out is expected for March 2022. Analyses will be completed in December 2022.

\section{Patient and public involvement}

Patients from different oncological support groups were involved during the preparation of the study. The questionnaires and patient informational materials on $\mathrm{CIH}$ used in the study were developed in cooperation with support groups. Given the nature of our naturalistically controlled trial, the public and media were addressed actively with information on the project, and lectures on $\mathrm{CIH}$ were held on patient days etc., in order to achieve our recruitment goal.

\section{ETHICAL CONSIDERATIONS, DATA PROTECTION ASPECTS AND DISSEMINATION}

The study has been approved by the appropriate Institutional Ethical Committee of the participating medical faculties (Freiburg, Heidelberg, Tuebingen, Ulm), No. 658/2019BO1.

Before study entry, all study participants are comprehensively informed about the project and a written declaration of consent for participation is obtained. Access to the new form of care is open to all patients, regardless of their particular statutory health insurance company. There are no additional costs or disadvantages for the participating patients. Patients' participation is voluntary. Participants will be informed verbally and in writing about the nature and scope of the planned procedure before the start of the study. Given informed consent, including the processing of patients' data, documented by signing the consent form, can be withdrawn at any time and without giving reasons. Disclosure takes place in pseudonymised form for the analysis. The names of the participating patients and all other confidential information are subject to medical confidentiality and the provisions of the European General Data Protection Regulation of 25 May 2018.

A comprehensive data protection concept with a data set prescription of the routine data was developed for the handling, transfer, and analysis of all data within the project. Contracts of data protection and for order processing were concluded with the partners. The partners are guided by the applicable standard for research projects and by the applicable data protection regulations. Approval according to $\$ 75$ SGB 5 for the transfer of social data for research was obtained from the AOK Baden-Wuerttemberg.
After completion of the analyses, the data will be made available on reasonable request in anonymised form in accordance with the institutional regulations and the General Data Protection Regulation (exception is the health insurance data of the reference group).

The study results will be presented to academic audiences through publication in peer-reviewed journals and presentations at national and international conferences. The results will also be disseminated in the community. The study is being conducted in collaboration with a health insurance company. The process evaluation will also include the identification of significant barriers and facilitating factors for implementation. If the evaluation of the project is successful, a transfer into standard care is planned.

\section{Author affiliations}

${ }^{1}$ Institute for General Practice and Interprofessional Care, University Hospital Tübingen, Tübingen, Germany

${ }^{2}$ Institute for Health Sciences, Department of Nursing Science, University Hospital Tübingen, Tübingen, Germany

${ }^{3}$ Institute for Clinical Epidemiology and Applied Biostatistics, University Hospital Tübingen, Tübingen, Germany

${ }^{4}$ Department of General Practice and Health Services Reseach, University Hospital Heidelberg, Heidelberg, Germany

${ }^{5}$ Department of Internal Medicine, Division of Pneumology, Paracelsus Medical University, Klinikum Nurnberg, Nurnberg, Germany

${ }^{6}$ aQua Institute for Applied Quality Improvement and Research in Health Care, Goettingen, Germany

${ }^{7}$ Department of Integrative Medicine, Faculty of Medicine, University Hospital Ulm, Ulm, Germany

${ }^{8}$ Department of Medicine I, Faculty of Medicine, University Hospital Freiburg, Freiburg, Germany

${ }^{9}$ Department of Medical Oncology, National Centre for Tumor Diseases, University Hospital Heidelberg, Heidelberg, Germany

${ }^{10}$ Fachbereich Integriertes Leistungsmanagement, AOK Baden-Württemberg, Stuttgart, Germany

Acknowledgements The authors gratefully thank native English speaker Hannah Fuhr for reviewing this manuscript. We acknowledge support by Open Access Publishing Fund of University of Tübingen.

Collaborators The CCC-Integrativ study group: A Schmitt, B Kröger, B Noack, B Wattenberg, E Kaschdailewitsch, H Mauch, K Gauß, K Harder, L Lohmüller, Institute for General Practice and Interprofessional Care, University Hospital and Faculty of Medicine Tuebingen, Germany; A Seckinger, D Zips, K Müller, Comprehensive Cancer Center Tübingen-Stuttgart, Germany; E Winkler, M Busacker-Scharpff, M Hoffmann, M Krug, M Reuter, S Eismann, Department of Medical Oncology, National Centre for Tumor Diseases, Heidelberg, Germany; A Kestler, B Leicht, C Nagat, C Raff, F Rapp, K Kraus, W Kmietschak, Department of Integrative Medicine, University Hospital and Faculty of Medicine Ulm, Germany; A Battran, A Frohn, B Held, C Perinchery, C Pfister-Jimenéz, Department of Medicine I, Medical Centre University of Freiburg, Faculty of Medicine, University of Freiburg, Germany; H Dürsch, J Bossert, J Szecsenyi, M Wensing, Department of General Practice and Health Services Research, University Hospital Heidelberg, Germany; A Kaltenbach, U Boltenhagen, Department of Nursing Science, Institute for Health Sciences, University Hospital and Faculty of Medicine Tuebingen, Germany; A Behzad, A Leppert. B Stein, Department of Internal Medicine, Division of Pneumology, Paracelsus Medical University, Klinikum Nuernberg, Germany; B Broge, C Witte, aQua Institute for Applied Quality Improvement and Research in Health Care, Goettingen, Germany; S Treffert, AOK Baden-Württemberg

Contributors SJ, CM, JV and RS conceptualised the design of the trial and interventions. All authors except PM, JF and KT-U were involved in the final elaboration of the intervention components. PM conducted the sample size calculation and contributed to the section on statistical methods. JV, DF and SJ wrote the first draft of this article. RS, CM, PM, NK, MH, JF, KK, HB, BG and KT-U critically revised it. JF contributed to the section on the health economic evaluation. All authors read and approved the final version of the manuscript. 
Funding This work was supported by the Innovationsfonds of the Federal Joint Committee 2019-2022 Grant number: 01NVF18004.

Competing interests None declared.

Patient and public involvement Patients and/or the public were involved in the design, or conduct, or reporting, or dissemination plans of this research. Refer to the Methods section for further details.

\section{Patient consent for publication Not applicable.}

Provenance and peer review Not commissioned; externally peer reviewed.

Open access This is an open access article distributed in accordance with the Creative Commons Attribution Non Commercial (CC BY-NC 4.0) license, which permits others to distribute, remix, adapt, build upon this work non-commercially, and license their derivative works on different terms, provided the original work is properly cited, appropriate credit is given, any changes made indicated, and the use is non-commercial. See: http://creativecommons.org/licenses/by-nc/4.0/.

ORCID iD

Jan Valentini http://orcid.org/0000-0003-4870-1853

\section{REFERENCES}

1 Horneber M, Bueschel G, Dennert G, et al. How many cancer patients use complementary and alternative medicine: a systematic review and metaanalysis. Integr Cancer Ther 2012;11:187-203.

2 Boon HS, Olatunde F, Zick SM. Trends in complementary/alternative medicine use by breast cancer survivors: comparing survey data from 1998 and 2005. BMC Womens Health 2007;7:4.

3 Leitlinienprogramm Onkologie der Arbeitsgemeinschaft der Wissenschaftlichen Medizinischen Fachgesellschaften e.V. (AWMF) DKeVDuDKD. Konsultationsfassung S3-Leitlinie Komplementärmedizin in der Behandlung von onkologischen PatientInnen, 2020.

4 Greenlee H, DuPont-Reyes MJ, Balneaves LG, et al. Clinical practice guidelines on the evidence-based use of integrative therapies during and after breast cancer treatment. CA Cancer J Clin 2017;67:194-232.

5 Lyman GH, Greenlee H, Bohlke K, et al. Integrative therapies during and after breast cancer treatment: ASCO endorsement of the SIO clinical practice guideline. J Clin Oncol 2018;36:2647-55.

6 Yeh GY, Chan CW, Wayne PM, et al. The impact of tai chi exercise on self-efficacy, social support, and Empowerment in heart failure: insights from a qualitative Sub-Study from a randomized controlled trial. PLoS One 2016;11:e0154678.

7 Hibbard JH, Mahoney ER, Stockard J, et al. Development and testing of a short form of the patient activation measure. Health Serv Res 2005:40:1918-30.

8 Nagel G, Schreiber D. Patientenkompetenz und Selfempowerment bei Patientinnen MIT Brustkrebs Im Frühstadium: ein Modell Der Kompetenzberatung. Deutsche Zeitschrift für Onkologie 2012;44:140-50.

9 Schaeffer D, Hurrlemann K, Bauer U. Nationaler Aktionsplan Gesundheitskompetenz. die Gesundheitskompetenz in Deutschland stärken. Berlin: KomPart, 2018.

10 Bravo P, Edwards A, Barr PJ, et al. Conceptualising patient empowerment: a mixed methods study. BMC Health Serv Res 2015;15:252:252.

11 Palumbo R. Examining the impacts of health literacy on healthcare costs. An evidence synthesis. Health Serv Manage Res 2017;30:197-212.

12 Alexander JA, Hearld LR, Mittler JN, et al. Patient-Physician role relationships and patient activation among individuals with chronic illness. Health Serv Res 2012;47:1201-23.

13 Ben-Arye E, Samuels N, Goldstein LH, et al. Potential risks associated with traditional herbal medicine use in cancer care: a study of middle Eastern oncology health care professionals. Cancer 2016;122:598-610.

14 Posadzki P, Watson LK, Ernst E. Adverse effects of herbal medicines: an overview of systematic reviews. Clin Med 2013;13:7-12.

15 Johnson SB, Park HS, Gross CP, et al. Complementary medicine, refusal of conventional cancer therapy, and survival among patients with curable cancers. JAMA Oncol 2018;4:1375-81.

16 Hayward RA, Asch SM, Hogan MM, et al. Sins of omission: getting too little medical care may be the greatest threat to patient safety. $J$ Gen Intern Med 2005;20:686-91.
17 Wardle Jonathan (Jon) Lee, Adams J. Indirect and non-health risks associated with complementary and alternative medicine use: an integrative review. Eur J Integr Med 2014;6:409-22.

18 Davis EL, Oh B, Butow PN, et al. Cancer patient disclosure and patient-doctor communication of complementary and alternative medicine use: a systematic review. Oncologist 2012;17:1475-81.

19 Sanford NN, Sher DJ, Ahn C, et al. Prevalence and nondisclosure of complementary and alternative medicine use in patients with cancer and cancer survivors in the United States. JAMA Oncol 2019;5:735-7.

20 Arslan C, Guler M. Alternative medicine usage among solid tumour patients receiving chemotherapy. Eur J Cancer Care 2017;26. doi:10.1111/ecc.12530. [Epub ahead of print: 2106 2016].

21 Hübner J. Chemotherapie. In: Hübner J, ed. Onkologie Interdisziplinär. Stuttgart: Schatauer, 2014: 54-61.

22 Shalom-Sharabi I, Samuels N, Lev E, et al. Impact of a complementary/integrative medicine program on the need for supportive cancer care-related medications. Support Care Cancer 2017;25:3181-90.

23 Coolbrandt A, Wildiers $\mathrm{H}$, Aertgeerts B, et al. Characteristics and effectiveness of complex nursing interventions aimed at reducing symptom burden in adult patients treated with chemotherapy: a systematic review of randomized controlled trials. Int J Nurs Stud 2014;51:495-510.

24 D'Egidio V, Sestili C, Mancino M, et al. Counseling interventions delivered in women with breast cancer to improve health-related quality of life: a systematic review. Qual Life Res 2017;26:2573-92.

25 Chambers SK, Hyde MK, Smith DP, et al. New challenges in Psycho-Oncology research III: a systematic review of psychological interventions for prostate cancer survivors and their partners: clinical and research implications. Psychooncology 2017;26:873-913.

26 Kreienberg R, Albert U, Follmann M. Interdisziplinäre S3-Leitlinie für Diagnostik, therapie und Nachsorge des Mammakarzinoms, 2012: 1-104.

27 Lederle M, Weltzien D, Bitzer EM. Führt die Steigerung von Gesundheitskompetenz und Selbstmanagement zu einer angemesseneren Inanspruchnahme gesundheitlicher Leistungen? Gesundheitswesen 2017;79:656-804.

28 Klein GE, Guethlin C. Information and training needs regarding complementary and alternative medicine: a cross-sectional study of cancer care providers in Germany. Integr Cancer Ther 2018;17:380-7.

29 Blödt S, Mittring N, Schützler L, et al. A consultation training program for physicians for communication about complementary medicine with breast cancer patients: a prospective, multi-center, clusterrandomized, mixed-method pilot study. BMC Cancer 2016;16:843.

30 Klafke N, Mahler C, von Hagens C, et al. A complex nursing intervention of complementary and alternative medicine (cam) to increase quality of life in patients with breast and gynecologic cancer undergoing chemotherapy: study protocol for a partially randomized patient preference trial. Trials 2015;16:51):51.

31 International Council of Nursing (ICN) Congress. Self-Efficacy in women experiencing complementary and alternative medicine in CONGO-study. Barcelona, Spain, 2017

32 National Research Center in Complementary and Alternative Medicine (NAFKAM) UTAUoN. National research center in complementary and alternative medicine (NAFKAM), uit the Arctic University of Norway, 2021. Available: https://cam-cancer.org/en [Accessed 11 Jun 2021].

33 Orchard C, Bursey S, Peterson L. Can workshops provide a way to enhance Patient/Client centered collaborative teams?: evidence of outcomes from TEAMc online facilitator training and team workshops?: evidence of outcomes from TEAMc online facilitator training and team workshops. International Journal of Practice-based Learning in Health and Social Care 2016;4:73-87.

34 Orchard C, 2007. Available: https://teamc.ca [Accessed 22 May 2021].

35 Brenk-Franz K, Hibbard JH, Herrmann WJ, et al. Validation of the German version of the patient activation measure 13 (PAM13-D) in an international multicentre study of primary care patients. PLoS One 2013;8:e74786.

36 Zill JM, Dwinger S, Kriston L, et al. Psychometric evaluation of the German version of the patient activation measure (PAM13). BMC Public Health 2013;13:1027.

37 Jansen F, Coupé VMH, Eerenstein SEJ, et al. Costs from a healthcare and societal perspective among cancer patients after total laryngectomy: are they related to patient activation? Support Care Cancer 2018;26:1221-31.

38 Rademakers J, Maindal HT, Steinsbekk A, et al. Patient activation in Europe: an international comparison of psychometric properties and 
patients' scores on the short form patient activation measure (PAM13). BMC Health Serv Res 2016;16:570.

39 Bates JS, Auten J, Sketch MR, et al. Patient engagement in first cycle comprehensive chemotherapy consultation pharmacist services and impact on patient activation. $J$ Oncol Pharm Pract 2019;25:896-902.

40 Harris PA, Taylor R, Thielke R, et al. Research electronic data capture (REDCap)--a metadata-driven methodology and workflow process for providing translational research informatics support. $J$ Biomed Inform 2009;42:377-81.

41 Kirk MA, Kelley C, Yankey N, et al. A systematic review of the use of the consolidated framework for implementation research. Implement Sci 2016;11:72.

42 Weiss FD, Mewes R, Rief W, et al. HCU-Q: Entwicklung eines Fragebogens Zur Erfassung Der Inanspruchnahme medizinischer Gesundheitsleistungen. Verhaltenstherapie 2018;28:15-24. 